\title{
Implementation and Analysis of Social Network Graph in Interpersonal Network
}

\author{
Akshay Tripathia1, Ankush Kumar Gaura2, Sweta Sria3 \\ Computer Science and Engineering, Kanpur Institute of Technology \\ Kanpur, India \\ 1akshaytripathi813@gmail.com (Corresponding author) \\ 2ankushkumardddm@gmail.com \\ 3er.swetasri@gmail.com
}

\begin{abstract}
Social graph describes the graphical model of users and how they are related to each other online. Social network consists of a set of nodes (sometimes referred to as actors or vertices in graph theory) connected via some type of relations which are known as edges. Actors are the smallest unit of the network. It can be Persons, Organizations, and Families etc. Relations can be of many types such as directed, undirected, and weighted. Social network analysis consists of two phases. One is data collection phase and another is analysis phase. Data is collected with the help of surveys, Social sites such as face book, Linkedln. We first input the user information in form of two dimensional matrices. Then we construct a graph based on the relationships among users from adjacency matrix. We can draw a directed graph or a simple graph based on the user input information from adjacency matrix. After analyzing the graph properties based on degree of node, centrality and other parameters we will give effective solution. There are many applications of analyzing social network for example examine a network of farm animals, to analyze how disease spread from one cow to another, discover emergent communities of interest among faculty at various universities, Some public sector uses include development of leader engagement strategies, analysis of individual and group engagement and media use, and community-based problem solving etc. Social network analysis is used widely in the social and behavioral sciences, as well as in economics, marketing, and industrial engineering. The social network perspective focuses on the relationships among social entities and is an important addition to standard social and behavioral research which is primarily concerned with attributes of the social units.
\end{abstract}

Keywords:

Adjacency matrix, Centrality, Degree, Edges, Social entity

\section{Introduction}

The Social network is a network of social interactionsand personal relationships. Social network can be defined as a social structure in which information of entities (users, organizations etc.) reside in form of text, databases, and communication systems. A dedicated website or other applications which enables users to communicate with each other by posting information, messages, images etc. is a kind of social network. A Social network is represented by using Graphs and Matrices. In Social graph construction and network analysis approach, the main task is to input the user information in form of 2D matrices known as adjacency matrices and constructing a graph from 2D matrices and then analyzing the graph based on its various properties. Social network offers a platform to people for sharing knowledge, thoughts or opinions and more often to maintain societal relationship. As shown in Fig. 1, analytically, these can observe as large graphs in which the users are represented as nodes and relationships between nodes are depicted as edges between them. The edge may or may not be directed depending on the structure of the social network and also an edge between two nodes depicts that the connected nodes have already sharedsome information as an alternative representation, adjacency matrix delineated in table 1 also represents Fig 1. 
Table 1: Adjacency Matrix representation of Social Network

\begin{tabular}{lccccccc}
\hline Actor/Users & Ramesh & Anubhav & Mohan & Dinesh & Sarita & Saroj & Bhanu \\
\hline Ramesh & - & 1 & 0 & 0 & 1 & 1 & 0 \\
Anubhav & 1 & $\ldots$ & 0 & 0 & 1 & 0 & 0 \\
Mohan & 0 & 0 & - & 0 & 0 & 1 & 1 \\
Dinesh & 0 & 0 & 0 & $-\ldots$ & 1 & 0 & 0 \\
Sarita & 1 & 1 & 0 & 1 & $\ldots$ & 0 & 0 \\
Saroj & 1 & 0 & 1 & 0 & 0 & $\ldots \ldots$ & 1 \\
Bhanu & 0 & 0 & 1 & 0 & 0 & 1 & - \\
\hline
\end{tabular}

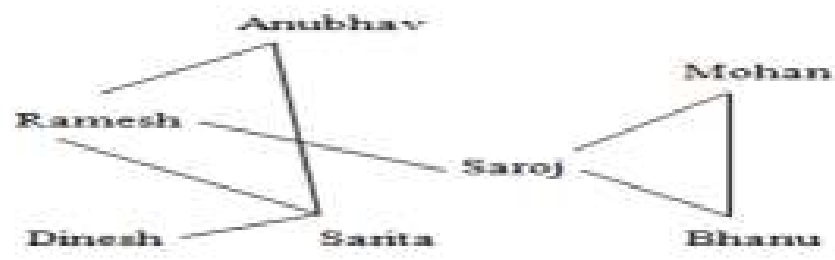

Figure 1. A Graphical representation of Social Network

\section{Literature Review}

A recent study has shown the advantages and disadvantages of facebook utilizing university students. A group of students responded to a survey containing advantages and disadvantages of facebook, and yielded interesting results. The study did not focus on students' academic performance, but took the issue as a general view of social media influence. Social networks are becoming major tools for education, and entertainment. The human nature is keen on interacting with people and finding common areas and interests. In education, two streams are prevailing: the use of social networks as a tool supporting activities deemed important for the purpose of educational institutions, instructors, and students. The second stream is the bad influence social network inflicts on students behaviors and time management. This study will explore the relationship between performance and $\mathrm{SN}$ use. The following sections will review the literature related to the two streams and the performance issue related to students

\section{General Properties of Social Graph}

Social network analysis is done to find mapping and measuring of relationships and flows between people, focusing on uncovered patterns of people's interaction, a set of methods for the investigation of relational aspects of social structures. After the construction of graph from adjacency matrix the main task is to now analyze the graph based on various properties of graph i.e. degree of the node in graph, geodesic distance and diameter, centrality, maximum flow etc. Network Visualization is shown as in Fig 2. 


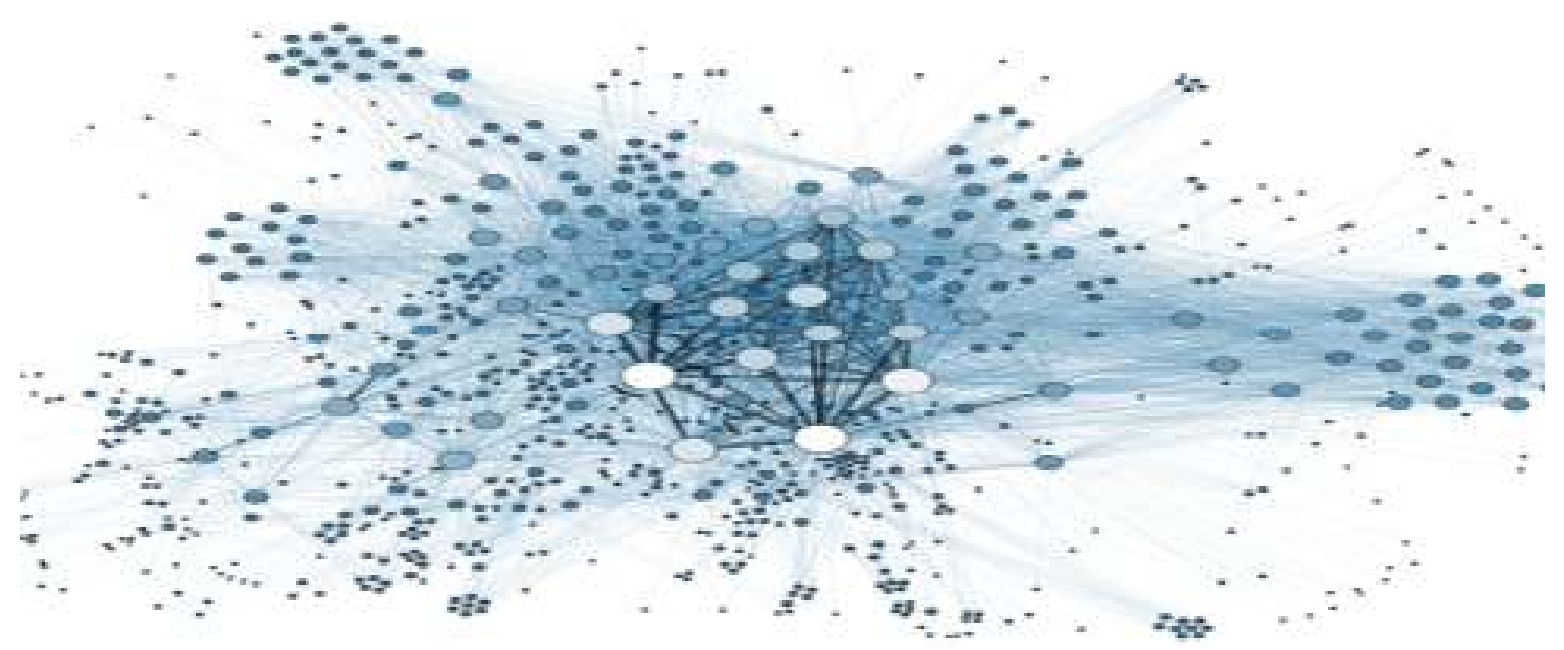

Figure 2. Network Visualization of Social Graph

\subsection{Degree}

Degree of a node is the number of edges connecting this node with other nodes .If graph is directed then a node has in-degree defined as number of incoming edges and out degree defined as number of outgoing edges.

\subsection{Geodesic Distance and Diameter}

For both directed and undirected graph, the geodesic distance is the number of edges in the shortest possible walk from one actor to another. Geodesic distance is widely used in network analysis. It determines the size of network.

\subsection{Cliques or Subgroups}

To understand that how the network is likely to behave, it is necessary to partition the actors into cliques or sub-groups. A clique is simply a sub-graph in which all nodes are more closely tied to each other. If the sub graph is complete graph there exists every possible tie among nodes then it is called maximal clique.

\subsection{Maximum Flow}

Reach ability of an actor to another depends on the number of factors in neighborhood that lead to the target. If an actor $A$ has to send a message to another actor $B$ and there is only one actor $C$ to whom $A$ can send message then $A$ has a weak connectivity with $B$ even if $C$ has many ways to reach $B$. On the other hand if $A$ can send message (destined to $B$ ) to four intermediate actor then connection between $A$ and $B$ is stronger. This flow shows that the strength of a tie between two actors depends on the weakest link in the chain of connections.

\subsection{Centrality}

Centrality is a measure that quantifies the influence of an actor in the network. It is normally assumed that central people have more influence than people who are less central in the network. Centrality can be measured by various terms such as Degree Centrality, Closeness Centrality, and betweenness centrality. 


\subsubsection{Degree Centrality}

It is known as number of ties for one actor. Centrality of a node depends on the number of nodes attached to it directly. For a network with g nodes the degree centrality attached to it directly and $d(n)$ is the number of nodes adjacent to it.

$$
\mathrm{cd}(\mathrm{n})=\mathrm{d}(\mathrm{n}) /(\mathrm{g}-1)
$$

\subsubsection{Betweenness Centrality}

Two nodes which are not adjacent may also influence each other significantly through other nodes in the network. Betweenness centrality measure highlights those nodes that fall in the connecting path of many nodes. For a network with g nodes, the betweenness centrality for node.

$$
\mathrm{C}_{\mathrm{b}}(\mathrm{x})=\sum_{\mathrm{j}<k} \mathrm{~g}_{\mathrm{jk}}\left(\mathrm{n}_{\mathrm{i}}\right) / \mathrm{g}_{\mathrm{jk}}
$$

Here, $g$ is the number of the shortest paths linking two nodes in the network $g(n)$ is the number of shortest path linking two nodes that go through the node $n$.

\subsubsection{Closeness Centrality}

An actor is considered important if he/she is relatively close to all other actors. It considers the sum of geodesic distance between a given node and rest. For the network with g nodes, the closeness centrality of node $n(i)$ is defined as following,

$$
\mathrm{C}_{\mathrm{c}}\left(\mathrm{n}_{\mathrm{i}}\right)=\left[\sum_{\mathrm{j}-1}^{\mathrm{g}} \mathrm{d}\left(\mathrm{n}_{\mathrm{i}}-\mathrm{n}_{\mathrm{j}}\right)\right]^{-1}
$$

Where, $d(n(i)-n(j))$ is the number of edges in the geodesic linking $n(i)$ and $n(j)$.

\section{Aims and Objective of Social Network Analysis}

The main objective of Social Network Analysis process is given as-

a) Collecting information about relationships within a defined group or network of people.

- Identifying the target network (e.g. team, group, and department).

- Formulating hypotheses and questions

- Outlining and clarifying objectives and the scope of analysis.

- Interviewing individuals in the network to identify relationships and knowledge flows.

- Determining the level of reporting required.

- Developing a survey methodology and the questionnaire.

b) Mapping out the network visually: mapping responses either manually or by using a software tool designed for the purpose.

c) Generating a baseline through the analysis of data from the survey responses.

d) Using this baseline for planning and prioritizing changes and interventions to improve social connections and knowledge flows within the group or network.

\section{Need and Application of Social Network Analysis}

Social network analysis methods facilitate some useful concept for addressing many levels of large social networks. At the analysis level social networks are seen as graph having nodes representing actors and edges as links between them. Therefore structural and statistical properties of graphs can be directly used to draw useful inferences as the outcome of the analysis. There are many applications of analyzing social network. For example, examining network of farm animals ,to analyze how disease spread from one cow to another, discover emergent communities of interest among faculty at various institutions, some public sector uses include 
development of leader engagement strategies, analysis of individual and group engagement and media use, and community based problem solving etc.

\section{Conclusion}

Social networks are becoming an integral part of people's lives. Students are spending much time on social media and are considered the largest category that uses such application. Social Network Analysis is an emerging technique for the analysis of relations data. Applications in the field of business and economics.

$>$ Interpersonal relations.

$>$ Inter organizational relations.

> Marketing applications: product and customer network.

Future research is encouraged to improve the instrument used and to reshape the dimensions that constitute the influence of face book on students' performance. A more comprehensive model might serve the purpose of research and conceptualize the issue related to performance. Also, a larger sample might serve better the large item size. Finally, this study is confined to the sample used; a different sample (males, other school levels, university students, or other demographic factors) might improve our understanding of this area.

\section{References}

[1] Wasserman, Stanley; Faust, Katherine (1994). "Social Network Analysis in the Social and Behavioural Sciences". Social Network Analysis: Methods and Applications. Cambridge University.

[2] Freeman, Linton C.; Wellman, Barry (1995). "A note on the ancestoral Toronto home of social network analysis". Connection.

[3] Levy, Judith and Bernice Pescosolido ( 2002). Social Networks and Health. Boston, MA: JAI Press.

[4] L. Akoglu and C. Faloutsos, "Event Detection in Time Series of Mobile Communication Graphs," 27th Army Science Conference, December 2010.

[5] M. Lahiri and T. Berger-Wolf, "Mining Periodic Behaviour in Dynamic Social Networks," Proceedings of the 8th IEEE International Conference on Data Mining, 2008, pp. 373-382.

[6] K. Carley, "Dynamic Network Analysis," Dynamic Social Network Modelling and Analysis:

[7] M. Rosvall and C.T. Bergstrom, "Maps of Random Walks on Complex Networks Reveal Community Structure," Proceedings of the National Academy of Sciences, vol. 105, no. 4, 2008, pp. 1118-1123

[8] J. Shi and J. Malik, "Normalized Cuts and Image Segmentation," IEEE Transactions on Pattern Analysis and Machine Intelligence, vol. 22, no. 8, 2000, pp. 888-905. 